\title{
Are neurological complications of monoclonal gammopathy of undetermined significance underestimated?
}

\author{
Normann Steiner ${ }^{1}$, Angelika Schwärzler ${ }^{1}$, Georg Göbel $^{3}$, Wolfgang Löscher ${ }^{2}$, Julia \\ Wanschitz ${ }^{2, *}$, Eberhard Gunsilius ${ }^{1, *}$ \\ ${ }^{1}$ Department of Internal Medicine V (Hematology and Medical Oncology), Medical University of Innsbruck, A-6020 Innsbruck, \\ Austria \\ ${ }^{2}$ Department of Neurology, Medical University of Innsbruck, A-6020 Innsbruck, Austria \\ ${ }^{3}$ Department of Medical Statistics, Informatics and Health Economics, Medical University of Innsbruck, A-6020 Innsbruck, \\ Austria \\ *These authors have contributed equally to this work \\ Correspondence to: Eberhard Gunsilius, email: eberhard.gunsilius@i-med.ac.at \\ Keywords: monoclonal gammopathy of undetermined significance, MGUS, MGUS associated neuropathy, multiple myeloma \\ Received: June 21, 2016 \\ Accepted: November 21, 2016 \\ Published: December 10, 2016
}

\section{ABSTRACT}

Objectives: Monoclonal gammopathy of undetermined significance (MGUS) is a premalignancy preceding multiple myeloma (MM) or related disorders. Neurological symptoms caused by the monoclonal immunoglobulins or free light-chains are often associated with a high morbidity. We analyzed the prevalence of neuropathy, clinical features and the long-term outcome in 223 patients (pts.) with MGUS.

Patients and Methods: Between 1/2005 and 3/2015, 223 adult pts. with MGUS were identified in our database.

Results: In36/223 pts. (16\%) a neuropathy was diagnosed (MGUS associated neuropathy, MGUS-N). 20 pts. (55\%) had a distal symmetric axonal neuropathy, 10 pts. $(28 \%)$ had a chronic inflammatory demyelinating polyneuropathy and 6 pts $(17 \%)$ a distal acquired demyelinating symmetric polyneuropathy. In MGUS-NN (without neuropathy) and in MGUS-N, progression to smoldering MM, MM or Waldenstrom's macroglobulinemia (WM) occurred in $17 \%$ of the pts. The Immunoglobulin subtype was predominantly IgG in MGUS-NN and IgM in MGUS-N and $\geq 5.5 \%$ plasma cells in the bone-marrow predicted progression to MM and AL-amyloidosis in MGUS-NN and to WM in MGUS-N $(p<0.05)$.

Conclusion: Due to the substantial prevalence of neuropathies, MGUS pts. should be monitored carefully and referred to a specialized center if neurological symptoms occur.

\section{INTRODUCTION}

Monoclonal gammopathy of undetermined significance (MGUS) is a premalignant disorder with a $0.5-1.5 \%$ per year risk of progression to multiple myeloma (MM) or other related hematological malignancies $[1,2]$. According to the International Myeloma Working Group (IMWG), MGUS is characterized by a monoclonal (M)protein in the serum of $<30 \mathrm{~g} / \mathrm{l}$, a clonal plasma cell count in the bone marrow of $<10 \%$, and the absence of clinical symptoms [3]. Risk factors for a progression include an M-protein $>15 \mathrm{~g} / 1$, an abnormal ratio of free kappa $(\kappa)$ and lambda $(\lambda)$ light chains, and the non-IgG isotype [4].

MGUS associated neuropathies (MGUS-N) are heterogeneous with respect to the clinical presentation and the underlying pathophysiology and can be caused by deposition of immunoglobulins or amyloid as well as through the interaction with specific antigens on peripheral nerves. Although the prevalence of neuropathy among MGUS patients (pts.) varies considerably in the literature and the identification often depends on patient selection and diagnostic procedures, it is estimated at about $17 \%$ [5-7]. Vice versa, 5-10\% of pts. investigated for neuropathy have a monoclonal gammopathy [8].

There are three major forms of neuropathy in paraproteinemic disorders: axonal sensory-motor neuropathy, chronic inflammatory demyelinating polyneuropathy (CIDP), and distal acquired demyelinating symmetric (DADS) polyneuropathy. Axonal neuropathy usually presents with sensory symptoms (paresthesia, 
dysesthesia, anesthesia, neuropathic pain) of distal lower limbs and slowly evolving motor weakness in a lengthdependent fashion. It may be associated with $\mathrm{IgG} / \mathrm{A} / \mathrm{M}$ MGUS, but the causal link between the serum paraprotein and axonal nerve damage remains elusive in many cases, although severe pain and autonomic dysfunction may raise the suspicion of amyloidosis [6]. In the demyelinating entities CIDP and DADS a causal relationship with monoclonal gammopathy is considered likely [6,9]. CIDP is a relapsing or progressive immune mediated neuropathy with proximal and distal weakness and sensory deficits of upper and lower limbs and 22-30\% of CIDP pts. are described to have MGUS, commonly IgG or IgA subtypes [10-12]. DADS neuropathy is characterized by predominant distal sensory impairment, ataxia and often tremor, but little or no weakness and has a close association with IgM kappa monoclonal gammopathy that is present in about two-thirds of pts. [13]. In 50-67\% of these pts. the IgM monoclonal protein binds to myelin-associated-protein (MAG) $[13,14]$ causing a characteristic widening of myelin lamellae in nerve biopsies [15]. Despite potent agents in the treatment of pts. with MGUS associated neuropathies, e.g. immunomodulatory agents, plasmapheresis or monoclonal antibodies, some pts. may still present with a high morbidity [9].

The aim of this retrospective single center analysis was to describe the prevalence of neurological manifestations in MGUS pts. and to compare clinical features and risk factors for disease progression in MGUS pts. with and without neuropathy.

\section{RESULTS}

\section{Patient characteristics}

223 pts. fulfilled the criteria for MGUS according to the International Myeloma Working Group (IMWG) criteria, thereof 187 pts. had a MGUS without (MGUS$\mathrm{NN} ; 84 \%$ ) and 36 showed a MGUS associated with neuropathy (MGUS-N; 16\%). Table 1 summarizes demographic data and laboratory features of MGUS-NN and MGUS-N pts.

Median age at diagnosis was 68 years (range 26-97 years) in the MGUS-NN group and 64 years (range 4282 years) in the MGUS-N group, respectively. Sex ratio was similar in MGUS-NN pts. (female $n=92,49 \%$; male $\mathrm{n}=95,51 \%$ ), while in the MGUS-N group significantly more pts. were male (female $n=7,19 \%$; male $n=29,81 \%$; $\mathrm{p}<0.05$ ). In the MGUS-NN cohort more IgG isotype was present than in MGUS-N pts. ( $n=137,74 \%$ vs. $n=17,47 \%$; $\mathrm{p}<0.05$ ), whereas more MGUS-N pts. had an IgM isotype $(\mathrm{n}=13,36 \%$ vs. $\mathrm{n}=27,14 \%$ in MGUS-NN; $\mathrm{p}<0.05)$. No significant differences in the prevalence of kappa $(\kappa)$ or lambda $(\lambda)$ light chains were observed. Concerning other laboratory findings, significantly more MGUS-NN pts. had anemia ( $\mathrm{n}=64,34 \%$ vs. $\mathrm{n}=514 \%$ in MGUS-N; $\mathrm{p}<0.05$ ).
More MGUS-NN pts. had renal dysfunction ( $\mathrm{n}=41,22 \%$ vs. $\mathrm{n}=5,14 \%$ in MGUS-N; $\mathrm{p}>0.05$ ), elevated calcium levels ( $\mathrm{n}=6,3 \%$ vs. $0 \%$ in MGUS-N; $\mathrm{p}>0.05$ ), elevated $\beta 2$-microglobulin levels ( $\mathrm{n}=68,43 \%$ vs. $\mathrm{n}=12,39 \%$ in MGUS-N; $\mathrm{p}>0.05)$, higher levels of lactat dehydrogenase $(\mathrm{LDH})(\mathrm{n}=33,18 \%$ vs. $\mathrm{n}=4,11 \%$ in MGUS-N; $\mathrm{p}>0.05)$, and a higher incidence of pre- or coexisting second tumors $(\mathrm{n}=57,31 \%$ vs. $\mathrm{n}=8,22 \%$ in MGUS-N; $\mathrm{p}>0.05)$. In the total study population 17 pts. (11\%) with IgG-MGUS, 6 pts. (22\%) with IgA-MGUS, 13 pts. (31\%) with IgMMGUS had neuropathy, but none had biclonal MGUS.

In the MGUS-N group 20 pts. (55\%) had an axonal neuropathy, 10 pts. (28\%) a CIDP, and 6 pts (17\%) presented with a DADS polyneuropathy. Anti-myelinassociated glycoprotein (MAG) antibodies were identified in 4 pts. with IgM-MGUS and demyelinating neuropathy (3 DADS and 1 CIDP phenotype). Table 2 specifies clinical symptoms and severity of neuropathy, associations with immunoglobulin isotypes and treatment modalities in MGUS-N pts. Severity of neuropathy was graded by review of pt. records as mild (sensory symptoms and/or mild distal weakness without impairment of walking), moderate (weakness and/or ataxia interfering with walking) or severe (walking with aid or wheelchairbound).

\section{Clinical outcome in MGUS-NN and MGUS-N pts}

Figure 1 shows an overview of the study population with regard to the progression to $\mathrm{MM}$ or other lymphoproliferative disorders. A total of 38 pts. (17\%) progressed after a median time to progression of 48 months (range 2-276 months). 6 pts. progressed in the MGUS-N cohort, thereof 4 pts. to Waldenstrom's macroglobulinemia $(67 \%)$ and 2 pts. to MM (33\%). In the MGUS-NN cohort, a total of $32 \mathrm{pts}$. progressed, thereof more than half of the pts. to MM ( $\mathrm{n}=17 ; 53 \%), 6$ pts. to smoldering MM (19\%), 7 pts. to AL-amyloidosis (22\%), and 2 pts. progressed to Waldenstrom's macroglobulinemia (6\%). Comparing the two cohorts MGUS-NN and MGUS-N, the rate of progressions was identical in the two groups $(17 \%)$. However, significantly more progressions to Waldenstrom's macroglobulinemia were observed in MGUS-N pts. $(67 \%$ vs. $6 \% ; p<0.05)$, and significantly more progressions to MM and AL-amyloidosis were seen in the MGUS-NN cohort $(53 \%$ vs. $33 \%$ and $22 \%$ vs. $0 \%$, respectively; both $\mathrm{p}=<0.05)$. Information on pts. who had a disease progression is shown in Table 3.

\section{Survival and time to progression (TTP) in MGUS-NN and MGUS-N pts}

Median follow-up for the whole cohort $(n=223)$ was 79 months and evaluated patient-years were 1,595. Median overall survival (OS) for the total study population 
Table 1: Demographic data and laboratory features at diagnosis; comparison of the two cohorts MGUS-NN and MGUS-N

\begin{tabular}{|c|c|c|c|c|}
\hline \multirow{2}{*}{ Parameter } & \multicolumn{2}{|c|}{ MGUS-NN } & \multicolumn{2}{|c|}{ MGUS-N } \\
\hline & $\mathrm{N}=\mathbf{1 8 7}$ & $\%$ & $\mathbf{N}=36$ & $\%$ \\
\hline Median age (range), years & $68(26-97)$ & & $64,5(42-82)$ & \\
\hline \multicolumn{5}{|l|}{ Sex f/m } \\
\hline $\mathrm{F}$ & 92 & 49* & 7 & $19 *$ \\
\hline M & 95 & $51 *$ & 29 & 81* \\
\hline \multicolumn{5}{|l|}{ Ig heavy chain (serum) } \\
\hline IgG & 137 & $74 *$ & 17 & $47 *$ \\
\hline $\operatorname{IgM}$ & 27 & $14 *$ & 13 & $36 *$ \\
\hline IgA & 17 & 9 & 6 & 17 \\
\hline \multicolumn{5}{|l|}{ biclonal gammopathy } \\
\hline $\operatorname{IgG}+\operatorname{IgA}$ & 4 & 2 & 0 & 0 \\
\hline $\operatorname{IgM}+\operatorname{IgG}$ & 2 & 1 & 0 & 0 \\
\hline \multicolumn{5}{|l|}{ Ig light chain (serum) } \\
\hline Kappa & 106 & 57 & 21 & 53 \\
\hline Lambda & 62 & 33 & 16 & 39 \\
\hline Both & 10 & 5 & 2 & 5 \\
\hline Not measurable & 9 & 5 & 1 & 3 \\
\hline Total protein $>\mathrm{UNV}(8 \mathrm{~g} / \mathrm{dl})$ & 12 & 6 & 3 & 8 \\
\hline$\beta-2$ microglobulin $>\mathrm{UNV}$ & 68 & 43 & 12 & 39 \\
\hline $\mathrm{LDH}>\mathrm{UNV}$ & 33 & 18 & 4 & 11 \\
\hline Creatinine $\geq 1.3 \mathrm{mg} / \mathrm{dl}$ & 41 & 22 & 5 & 14 \\
\hline Serum calcium $>$ UNV & 6 & 3 & 0 & 0 \\
\hline Haemoglobin $\leq 12 \mathrm{~g} / \mathrm{dl}$ & 64 & $34 *$ & 5 & 14 * \\
\hline Platelets $<100,000 / \mathrm{mm}^{3}$ & 18 & 10 & 4 & 11 \\
\hline Osteolytic bone lesion & 6 & 3 & 1 & 3 \\
\hline
\end{tabular}

MGUS-NN, monoclonal gammopathy of undetermined significance without neuropathy; MGUS-N, monoclonal gammopathy of undetermined significance associated neuropathy; N, number of patients; Ig, Immunoglobulin; UNV, upper normal value; $\mathrm{LDH}$, lactate dehydrogenase.

* p-value $<0.05$.

was 255 months. In the progressed 38 pts. a progression rate in the first year of $26 \%$ was seen. The progression rate was $1 \%$ per year in the MGUS-N cohort and $0.5 \%$ per year in the MGUS-NN cohort. Median TTP in the cohort that progressed (MGUS-NN and MGUS-N; $\mathrm{n}=38$ ) was 48 months (95\% CI 20-76). TTP was longer in the MGUSNN group than in the MGUS-N cohort (median TTP 54 months, $95 \% \mathrm{CI}=30-78$ vs. median TTP 12 months, $95 \% \mathrm{CI}=8-16 ; \mathrm{p}>0.05)$. Kaplan-Meier plots on progression for the whole cohort $(\mathrm{n}=223)$ and stratified for MGUS-NN and MGUS-N are shown in Figure 2.

\section{Risk factors for disease progression}

Clinical data of the two cohorts, MGUS-NN and MGUS-N, were evaluated in terms of risk factors for disease progression.

Progressed pts. with MGUS-NN ( $\mathrm{n}=32)$ showed a significantly higher incidence of $\mathrm{IgG}$ isotype $(\mathrm{n}=25,78 \%$ vs. $\mathrm{n}=1,17 \%$ in MGUS-N; $\mathrm{p}<0.05)$, whereas MGUS-N pts. with progressive disease $(n=6)$ had significantly more IgM isotype $(n=4,67 \%$ vs. $n=2,6 \%$ in MGUS-NN; $\mathrm{p}<0.05$ ). Only male pts. progressed to MM or other related 
Table 2: Clinical characteristics of patients with MGUS associated neuropathy

\begin{tabular}{|c|c|c|c|}
\hline \multicolumn{4}{|c|}{ MGUS-N N=36 (100\%) } \\
\hline & axonal neuropathy & CIDP & DADS \\
\hline $\mathrm{N}(\%)$ & $20(55)$ & $10(28)$ & $6(17)$ \\
\hline \multicolumn{4}{|l|}{ Sex f/m } \\
\hline $\mathrm{f}(\%)$ & $5(14)$ & $2(6)$ & $0(0)$ \\
\hline $\mathrm{m}(\%)$ & $15(42)$ & $8(22)$ & $6(17)$ \\
\hline \multicolumn{4}{|l|}{ Ig isotype } \\
\hline $\operatorname{IgG}$ & $10(28)$ & 7 (19) & $0(0)$ \\
\hline $\operatorname{IgM}$ & $6(17)$ & $2(6)$ & $5(14)$ \\
\hline $\operatorname{IgA}$ & $4(11)$ & $1(3)$ & $1(3)$ \\
\hline \multicolumn{4}{|l|}{ Treatment } \\
\hline Steroids & $3(8)$ & $9(25)$ & $2(6)$ \\
\hline IVIG & $4(11)$ & $10(28)$ & $4(11)$ \\
\hline Rituximab & $2(6)$ & $3(8)$ & $3(8)$ \\
\hline Azathioprine & $0(0)$ & $2(6)$ & $0(0)$ \\
\hline Mycophenolate-Mofetil & $0(0)$ & $2(6)$ & $0(0)$ \\
\hline Lenalidomide & $0(0)$ & $1(3)$ & $1(3)$ \\
\hline Carfilzomib & $0(0)$ & $0(0)$ & $1(3)$ \\
\hline Plasmapheresis & $0(0)$ & $2(6)$ & $0(0)$ \\
\hline Pain therapy & $8(22)$ & $7(19)$ & $3(8)$ \\
\hline \multicolumn{4}{|l|}{ Pain } \\
\hline Yes & $10(28)$ & $9(25)$ & $4(11)$ \\
\hline No & $10(28)$ & $1(3)$ & $2(6)$ \\
\hline \multicolumn{4}{|l|}{ Symptoms of neuropathy } \\
\hline Motor, only & $3(8)$ & $0(0)$ & $0(0)$ \\
\hline Sensory, only & $8(22)$ & $0(0)$ & $1(3)$ \\
\hline Sensorimotor & $8(22)$ & $10(28)$ & $5(14)$ \\
\hline Tremor & $6(17)$ & $3(8)$ & $2(6)$ \\
\hline Not classified & $1(3)$ & $0(0)$ & $0(0)$ \\
\hline \multicolumn{4}{|l|}{ Severity of neuropathy } \\
\hline Mild & $2(6)$ & $1(3)$ & $1(3)$ \\
\hline Moderate & $1(3)$ & $4(11)$ & $2(6)$ \\
\hline Severe & $1(3)$ & $5(14)$ & $3(8)$ \\
\hline Not classified & $16(44)$ & $0(0)$ & $0(0)$ \\
\hline
\end{tabular}

DADS, distal acquired demyelinating symmetric neuropathy; CIDP, chronic inflammatory demyelinating polyneuropathy; Ig, Immunoglobulin; IVIG, Intravenous immunoglobulin. 
diseases in the MGUS-N group ( $n=6,100 \%$ vs. $n=15,47 \%$ in MGUS-NN; $\mathrm{p}<0.05)$.

12 pts. $(32 \%)$ of the progressed 38 pts. had second tumors, thereof 11 pts. (34\%) were in the MGUSNN group and 1 pt. (17\%) was in the MGUS-N group $(p>0.05) .4$ pts. had prostate cancer before diagnosis of MGUS, and all but two tumors developed before progression of MGUS.

Furthermore, in the total study population, pts. with a percentage of $\geq 5.5 \%$ plasma cells in the bone marrow had a significantly higher risk for progression than pts. with $<5.5 \%$ plasma cells in the bone marrow (progression risk $38 \%$ vs. $7 \%, \mathrm{p}<0.05$ ).

\section{DISCUSSION}

In this retrospective study, 223 MGUS pts. were evaluated in terms of neurological manifestations. 187 MGUS-NN pts. were compared to 36 MGUS-N pts. regarding clinical characteristics, disease progression and risk factors predicting progression to a malignant hematological disorder. The strengths of this study are the large number of included pts., all collected in the local database of our medical university, and the follow-up time of 10 years. Limitations existed in the partly incomplete documentation of pts.' data intended for the analysis. Moreover, pts. treated at a University Hospital might create a selection bias in favor of MGUS-N pts.

The prevalence of several clinical and laboratory features (immunoglobulins, anemia, renal dysfunction, elevated calcium level, elevated $\beta 2$-microglobulin, LDH level, incidence of second tumors, progression to different hematological diseases) differed between MGUS-NN and MGUS-N (Table 1). A significantly higher incidence of anemia was found in MGUS-NN. A potential explanation could be the significantly higher prevalence of $\mathrm{IgG}$ immunoglobulins in the MGUS-NN cohort, which can lead to monoclonal gammopathy of renal significance including chronic kidney disease with renal anemia $[16,17]$.

The annual risk of progression to $\mathrm{MM}$ in pts. with MGUS is about $1 \%$ [1]. The risk of progression to $\mathrm{MM}$

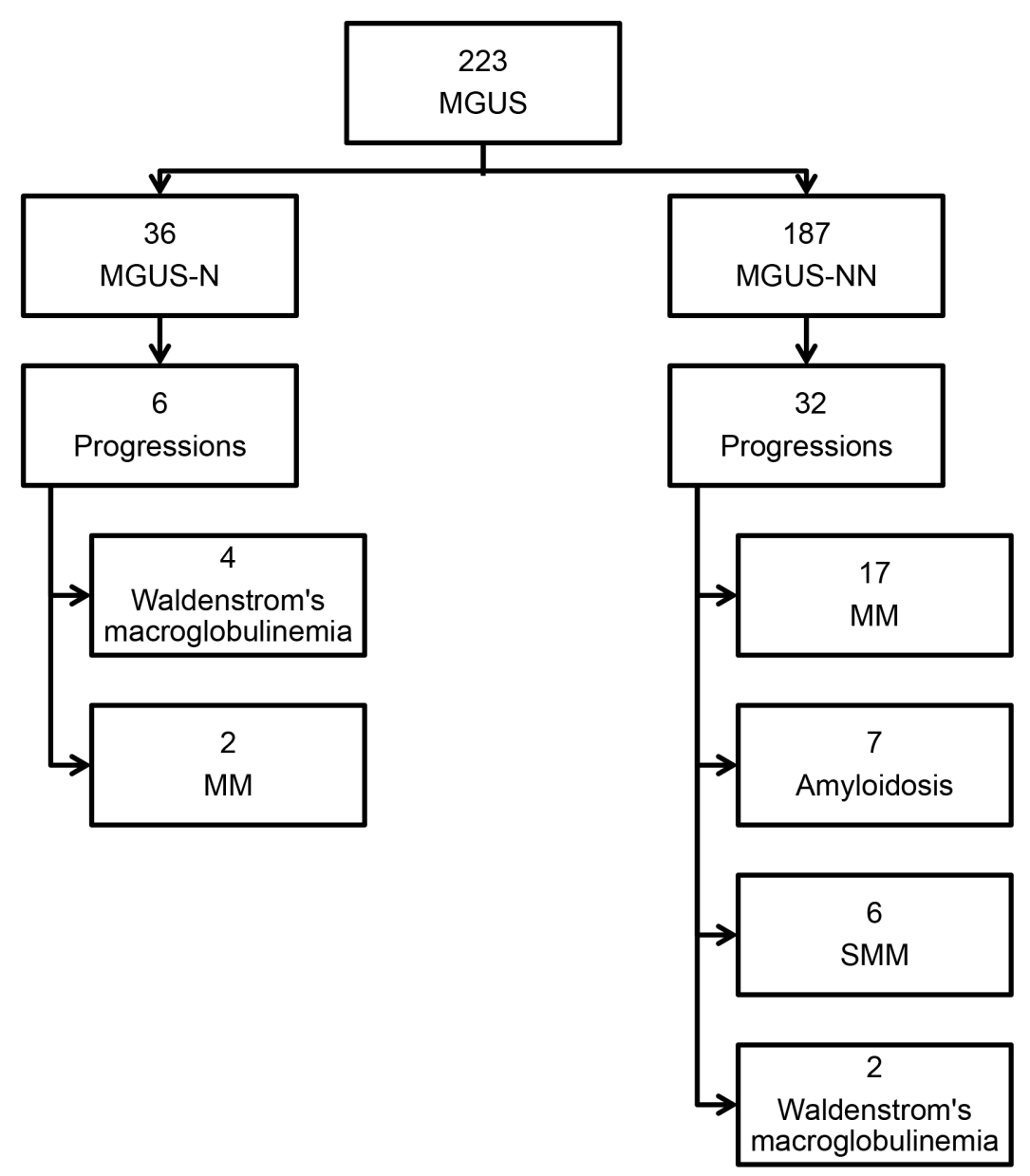

Figure 1: Study population overview. MGUS-NN, monoclonal gammopathy of undetermined significance without neuropathy; MGUS-N, monoclonal gammopathy of undetermined significance associated neuropathy; SMM, smoldering multiple myeloma; MM, multiple myeloma. 
Table 3: Characteristics of persons who progressed to SMM, MM, AL-amyloidosis or Waldenstrom's macroglobulinemia

\begin{tabular}{|c|c|c|c|c|c|c|c|c|}
\hline ID & Sex & Age & $\begin{array}{l}\text { M-protein and free } \\
\text { light chains(serum) }\end{array}$ & Neuropathy & Progression & TTP & 2nd Tumor & $\begin{array}{l}\text { LFU } \\
\text { status }\end{array}$ \\
\hline 1 & $\mathrm{~m}$ & 73 & $\operatorname{Ig} A \lambda$ & - & SMM & 48 & - & dead \\
\hline 2 & $\mathrm{f}$ & 43 & $\operatorname{IgG} \lambda$ & - & $\mathrm{MM}$ & 276 & & alive \\
\hline 3 & $\mathrm{f}$ & 66 & $\operatorname{IgG\kappa }$ & - & SMM & 58 & - & alive \\
\hline 4 & $\mathrm{~m}$ & 63 & $\operatorname{IgG\kappa }$ & - & AL-amyloidosis & 114 & - & \\
\hline 5 & $\mathrm{~m}$ & 66 & $\operatorname{IgM\kappa }$ & $\begin{array}{c}\text { Axonal } \\
\text { neuropathy }\end{array}$ & $\begin{array}{c}\text { Waldenstrom's } \\
\text { macroglobulinemia }\end{array}$ & 13 & - & alive \\
\hline 6 & $\mathrm{f}$ & 72 & $\operatorname{IgG\kappa }$ & - & $\mathrm{MM}$ & 159 & $\begin{array}{l}\text { Skin-CA } \\
\text { (squamous } \\
\text { cell CA) }\end{array}$ & alive \\
\hline 7 & $\mathrm{f}$ & 62 & IgGK & - & $\mathrm{MM}$ & 27 & - & alive \\
\hline 8 & $\mathrm{f}$ & 78 & $\operatorname{IgM\kappa }$ & - & $\begin{array}{c}\text { Waldenstrom's } \\
\text { macroglobulinemia }\end{array}$ & 106 & - & alive \\
\hline 9 & $\mathrm{~m}$ & 58 & $\operatorname{IgG} \lambda$ & - & MM & 63 & Meningeoma & alive \\
\hline 10 & $\mathrm{~m}$ & 66 & $\operatorname{IgG\kappa }$ & - & MM & 2 & - & dead \\
\hline 11 & $\mathrm{~m}$ & 74 & $\operatorname{IgA\kappa }$ & $\begin{array}{c}\text { Axonal } \\
\text { neuropathy }\end{array}$ & $\mathrm{MM}$ & 99 & - & dead \\
\hline 12 & $\mathrm{f}$ & 56 & $\operatorname{IgG} \lambda$ & - & AL-amyloidosis & 10 & - & dead \\
\hline 13 & $\mathrm{~m}$ & 61 & $\operatorname{IgM} \lambda$ & DADS & $\begin{array}{c}\text { Waldenstrom's } \\
\text { macroglobulinemia }\end{array}$ & 10 & - & alive \\
\hline 14 & $\mathrm{f}$ & 77 & $\operatorname{IgG} \lambda$ & - & $\mathrm{MM}$ & 35 & - & alive \\
\hline 15 & $\mathrm{~m}$ & 65 & $\operatorname{IgA\kappa }$ & - & $\mathrm{MM}$ & 112 & - & alive \\
\hline 16 & $\mathrm{~m}$ & 60 & $\operatorname{IgG\kappa }$ & - & AL-amyloidosis & 230 & - & dead \\
\hline 17 & $\mathrm{~m}$ & 78 & $\operatorname{IgG\kappa }$ & - & MM & 8 & Prostate-CA & alive \\
\hline 18 & $\mathrm{f}$ & 81 & $\operatorname{IgG} \lambda$ & - & $\mathrm{MM}$ & 19 & Meningeoma & alive \\
\hline 19 & $\mathrm{f}$ & 76 & $\operatorname{IgG} \lambda$ & - & MM & 68 & - & alive \\
\hline 20 & $\mathrm{~m}$ & 65 & $\operatorname{Ig} A \lambda$ & - & AL-amyloidosis & 4 & - & dead \\
\hline 21 & $\mathrm{f}$ & 49 & $\operatorname{IgG\kappa }$ & - & MM & 58 & - & dead \\
\hline 22 & $\mathrm{~m}$ & 75 & $\operatorname{IgM\kappa }$ & $\begin{array}{c}\text { Axonal } \\
\text { neuropathy }\end{array}$ & $\begin{array}{l}\text { Waldenstrom's } \\
\text { macroglobulinemia }\end{array}$ & 91 & $\begin{array}{l}\text { Urothelial-CA+ } \\
\text { Lung-CA }\end{array}$ & dead \\
\hline 23 & $\mathrm{~m}$ & 70 & $\operatorname{IgG\kappa }$ & - & SMM & 68 & - & alive \\
\hline 24 & $\mathrm{f}$ & 62 & $\operatorname{IgA\kappa }$ & - & $\mathrm{MM}$ & 34 & - & alive \\
\hline 25 & $\mathrm{~m}$ & 67 & $\operatorname{IgG} \lambda$ & - & AL-amyloidosis & 73 & Prostate-CA & alive \\
\hline 26 & $\mathrm{f}$ & 65 & $\operatorname{IgA\kappa }$ & - & SMM & 158 & - & alive \\
\hline 27 & $\mathrm{~m}$ & 65 & $\operatorname{IgG} \lambda$ & - & AL-amyloidosis & 35 & Prostate-CA & dead \\
\hline 28 & $\mathrm{f}$ & 85 & $\operatorname{IgG\kappa }$ & - & AL-amyloidosis & 35 & Urothelial-CA & dead \\
\hline 29 & $\mathrm{~m}$ & 61 & $\operatorname{IgG\kappa }$ & - & MM & 41 & Prostate-CA & alive \\
\hline 30 & $\mathrm{~m}$ & 66 & $\operatorname{IgG\kappa }$ & - & SMM & 9 & $\begin{array}{c}\text { Sezary- } \\
\text { Syndrome }\end{array}$ & dead \\
\hline
\end{tabular}

(Continued) 


\begin{tabular}{|c|c|c|c|c|c|c|c|c|}
\hline ID & Sex & Age & $\begin{array}{l}\text { M-protein and free } \\
\text { light chains(serum) }\end{array}$ & Neuropathy & Progression & TTP & 2nd Tumor & $\begin{array}{l}\text { LFU } \\
\text { status }\end{array}$ \\
\hline 31 & $\mathrm{~m}$ & 84 & $\operatorname{IgG\kappa }$ & - & SMM & 7 & - & dead \\
\hline 32 & $\mathrm{~m}$ & 66 & $\operatorname{IgG} \lambda$ & - & MM & 11 & - & alive \\
\hline 33 & $\mathrm{f}$ & 54 & $\operatorname{IgG} \lambda$ & - & $\mathrm{MM}$ & 54 & - & alive \\
\hline 34 & $\mathrm{f}$ & 66 & $\operatorname{IgG\kappa }$ & - & MM & 248 & Meningeoma & alive \\
\hline 35 & $\mathrm{f}$ & 58 & $\operatorname{IgM\kappa }$ & - & $\begin{array}{c}\text { Waldenstrom's } \\
\text { macroglobulinemia }\end{array}$ & 163 & - & alive \\
\hline 36 & $\mathrm{~m}$ & 61 & $\operatorname{IgG\kappa }$ & CIDP & MM & 12 & - & alive \\
\hline 37 & $\mathrm{f}$ & 48 & $\operatorname{IgG\kappa }+\lambda$ & - & MM & 272 & Gastric-CA & alive \\
\hline 38 & $\mathrm{~m}$ & 57 & $\operatorname{IgM\kappa }$ & DADS & $\begin{array}{l}\text { Waldenstrom's } \\
\text { macroglobulinemia }\end{array}$ & 3 & - & alive \\
\hline
\end{tabular}

TTP, time to progression in months; LFU, last follow-up; DADS, distal acquired demyelinating symmetric neuropathy; CIDP, chronic inflammatory demyelinating polyneuropathy; SMM, smoldering multiple myeloma; MM, multiple myeloma; CA, Carcinoma.

in pts. with light chain MGUS is $0.3 \%$ [2]. Our results confirmed these findings but differed between the two cohorts (progression rate $1 \%$ /year in the MGUS-N cohort vs. $0.5 \%$ /year in the MGUS-NN cohort), although the difference was not statistically significant. 38 of 223 pts. (17\%) progressed to MM or other related hematological diseases (Figure 1). It was shown that pts. affected by MGUS-NN, progressed significantly more often to smoldering MM, MM and AL-amyloidosis compared to the MGUS-N group. By contrast, more progressions to Waldenstrom's macroglobulinemia were seen in the MGUS-N group. In particular, the immunoglobulin isotype, the M-protein concentration and the free light chains ratio are known to be risk factors related to progression [1, 4, 18]. Recently, Sigurdardottir and coworkers demonstrated that regular follow-up of MGUS pts. may be associated with the outcome in MM [19]. Thus, the guidelines recommend annual monitoring in high risk MGUS pts. and follow-up in low risk MGUS pts. presenting with clinical symptoms [4]. As optional investigation, in IgM-MGUS with higher risk for progression into Waldenstrom's macroglobulinemia than non-IgM MGUS, an evaluation of lymphadenopathy and/ or spleen size by sonography could be done.

In our cohort, 36 pts. (16\%) with MGUS had an associated neuropathy. This finding is in line with the study of Nobile-Orazio et al., who described 17\% MGUS-N in their population (thereof $6 \%$ in IgG-MGUS, $14 \%$ in IgA-MGUS, and $31 \%$ in IgM-MGUS pts.) [5]. Axonal neuropathy was the most frequent entity among our pts. (55\% of MGUS-N) and most of these cases probably had a coincidental association with MGUS not requiring specific treatment except agents for neuropathic pain [9, 20]. Some pts. with $\operatorname{IgG}$ and $\operatorname{IgA}$ paraproteinaemic axonal neuropathy, however, may benefit from immunotherapy [21], as was seen in two pts. from our cohort; therefore these pts. should not be a priori excluded from these treatment options.

IgM-MGUS has the highest prevalence in monoclonal gammopathy associated with neuropathy, that usually manifests with distal symmetric sensorimotor and atactic features $[6,22,23]$. These findings (IgMMGUS-N in our total IgM cohort of 42 pts. was $31 \%$ ) and the results of Kristinsson et al., that MGUS of IgM type is associated with a higher progression risk to Waldenstrom's macroglobulinemia [24], are confirmed by our study. In our cohort, all 6 pts. who progressed to Waldenstrom's macroglobulinemia had previously IgM-MGUS-NN or IgM-MGUS-N, respectively (see Table 3). The MGUS-N group showed significantly more progressions to Waldenstrom's macroglobulinemia compared to the MGUS-NN group (4 of 6 progressed pts. in MGUS-N; $67 \%$ vs. 2 of 32 progressed pts. in MGUS$\mathrm{NN} ; 6 \% ; \mathrm{p}<0.05)$. The disease course of neuropathy due to IgM-MGUS with or without antibodies against MAG is highly variable [25] and studies assessing the efficacy of intravenous immunoglobulins [26,27] and Rituximab $[28,29]$ showed inconsistent results. In CIDP associated with monoclonal gammopathy, steroids, intravenous immunoglobulins and plasma exchanges represent the main therapeutic options as in idiopathic CIDP and 66$80 \%$ of pts. respond to one of these treatments [11, 30, 31]. On long-term follow-up, however, progression of functional deficits is greater in MGUS-CIDP pts. compared to idiopathic CIDP [32].

In our total study population, a threshold of $\geq 5.5 \%$ plasma cells in the bone marrow was found to be a strong predictor for disease progression. Although this 

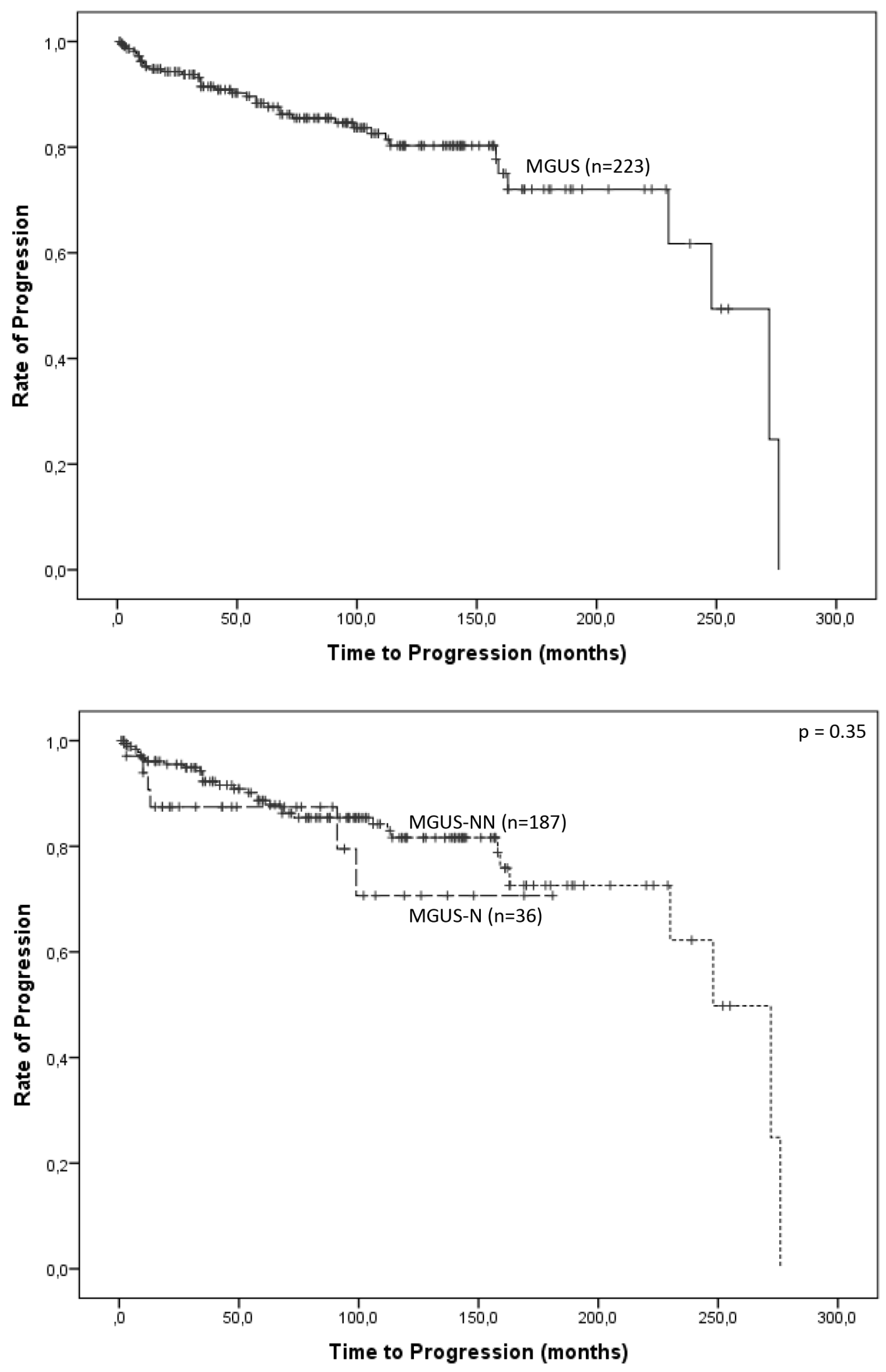

Figure 2: Kaplan-Meier plots on progression for the whole cohort $(n=223)$, and stratified for MGUS-NN and MGUS-N. 
was described previously [33, 34], nevertheless it is an important finding and should be evaluated in the future as a risk factor for disease progression. Hitherto, special molecular alterations and drivers for disease progression in MGUS and MM are still unknown, but it has been shown that changes in the bone marrow microenvironment develop early and consistently [35]. It might be suggested that clonal proliferation of plasma cells increases genetic instability with possible driver mutations in different subclones [36, 37]. The higher the clonal expansion, the higher the number of potential triggers. Therefore, in our opinion, for MGUS pts. with a percentage of $\geq 5.5 \%$ plasma cells in the bone marrow, a close-meshed monitoring to detect progression to $\mathrm{MM}$ or other related hematological disorders is recommended. However, a routine bone marrow examination in newly diagnosed MGUS pts. is not currently indicated.

In summary, in this study we analyzed the prevalence of neurological manifestations in MGUS pts. and demonstrated differences in clinical features and risk factors for disease progression in a large cohort of MGUS-NN and MGUS-N pts. In our study population, a considerable part of MGUS pts. (16\%) had a neuropathy. Peripheral neuropathy associated with monoclonal gammopathy is a complex problem in clinical practice. As MGUS pts. frequently are not referred to a specialized center, that may result in an underestimation of neurological symptoms and their complications or in a non-detection of a correlation with MGUS-N disease. Therefore, it is important that MGUS pts. are monitored carefully and referred to a specialized center if neurological symptoms occur, particularly those with progressive sensorimotor deficits and ataxia. Furthermore, due to the risk of progression to MM or other related diseases (17\% of the pts. in both groups), the important goal for MGUS pts. must be the detection of an early progression into MM and the prevention of complications. MGUS pts. should be considered for riskand-response stratified therapy monitoring even in terms of neurological manifestations, and an early supportive treatment should be conducted to improve their quality of life, as well as immunomodulatory and /or specific treatments in pts. with progressive neuropathies.

\section{PATIENTS AND METHODS}

Between January 2005 and March 2015, a total of 223 adult pts. aged between 26 and 97 years with MGUS according to the International Myeloma Working Group (IMWG) criteria [3], were identified by an exploration in our local database. Observation period in some pts. lasted over 10 years since the initial diagnosis was made before 2005. Sampling and evaluation of pts.' data was approved by the local institutional ethics committee (vote number: AN2015-0193 352/4.13) and was conducted in accordance with the principles of the Declaration of Helsinki. The study included pts.' data about the clinical presentation, physical examination and laboratory tests. Two cohorts, MGUS-NN and MGUS-N pts., were compared and were analyzed for disease progression. "Progression" is used in the whole paper as term for every change into other diseases. MGUS-N pts. were diagnosed by a consultant neurologist or referred to the neuromuscular unit of the Department of Neurology and classification of neuropathy was based on standard electrophysiological studies.

Statistical evaluation was performed using SPSS statistical software (version 20.0; SPSS Inc., Chicago, IL, USA). All tests for statistical significance were two-sided. Chi-squared test, unpaired t-test and survival analysis (Kaplan Meier curves, log-rank test) were used to identify differences between two groups. A p-value of $<0.05$ was considered as statistically significant.

\section{ACKNOWLEDGMENTS}

Author's contributions to this work: N Steiner, A Schwärzler and J Wanschitz identified the pts. from the local database. N Steiner and A Schwärzler performed the data analysis and interpretation. E Gunsilius, J Wanschitz, WN Löscher and N Steiner wrote the paper. Statistical evaluation was performed by $\mathrm{G}$ Göbel.

\section{CONFLICTS OF INTEREST}

The authors declare no financial conflicts of interest.

\section{REFERENCES}

1. Kyle RA, Durie BG, Rajkumar SV, Landgren O, Blade J, Merlini G, Kroger N, Einsele H, Vesole DH, Dimopoulos M, San Miguel J, Avet-Loiseau H, Hajek R, et al. Monoclonal gammopathy of undetermined significance (MGUS) and smoldering (asymptomatic) multiple myeloma: IMWG consensus perspectives risk factors for progression and guidelines for monitoring and management. Leukemia. 2010; 24: 1121-1127.

2. Dispenzieri A, Katzmann JA, Kyle RA, Larson DR, Melton LJ, 3rd, Colby CL, Therneau TM, Clark R, Kumar SK, Bradwell A, Fonseca R, Jelinek DF, Rajkumar SV, et al. Prevalence and risk of progression of light-chain monoclonal gammopathy of undetermined significance: a retrospective population-based cohort study. Lancet . 2010; 375: 1721-1728.

3. Group IMW. Criteria for the classification of monoclonal gammopathies, multiple myeloma and related disorders: a report of the International Myeloma Working Group. Br J Haematol. 2003; 121: 749-757.

4. www.onkopedia.com/de/onkopedia/guidelines/monoklonalegammopathie-unklarer-signifikanz-mgus/@@view/html/ index.html.

5. Nobile-Orazio E, Barbieri S, Baldini L, Marmiroli P, Carpo M, Premoselli S, Manfredini E, and Scarlato G. Peripheral 
neuropathy in monoclonal gammopathy of undetermined significance: prevalence and immunopathogenetic studies. Acta Neurol Scand. 1992; 85: 383-390.

6. Rajabally YA. Neuropathy and paraproteins: review of a complex association. Eur J Neurol. 2011; 18: 1291-1298.

7. Gosselin S, Kyle RA, and Dyck PJ. Neuropathy associated with monoclonal gammopathies of undetermined significance. Ann Neurol. 199; 30: 54-61.

8. Kelly JJ, Jr., Kyle RA, O'Brien PC, and Dyck PJ. Prevalence of monoclonal protein in peripheral neuropathy. Neurology. 1981; 31: 1480-1483.

9. Rison RA and Beydoun SR. Paraproteinemic neuropathy: a practical review. BMC Neurol. 2016; 16: 13.

10. Simmons Z, Albers JW, Bromberg MB, and Feldman EL. Presentation and initial clinical course in patients with chronic inflammatory demyelinating polyradiculoneuropathy: comparison of patients without and with monoclonal gammopathy. Neurology. 1993; 43: 2202-2209.

11. Gorson KC, Allam G, and Ropper AH. Chronic inflammatory demyelinating polyneuropathy: clinical features and response to treatment in 67 consecutive patients with and without a monoclonal gammopathy. Neurology. 1997; 48: 321-328.

12. Bromberg MB, Feldman EL, and Albers JW. Chronic inflammatory demyelinating polyradiculoneuropathy: comparison of patients with and without an associated monoclonal gammopathy. Neurology. 1992; 42: 1157-1163.

13. Katz JS, Saperstein DS, Gronseth G, Amato AA, and Barohn RJ. Distal acquired demyelinating symmetric neuropathy. Neurology. 2000; 54: 615-620.

14. Nobile-Orazio E, Manfredini E, Carpo M, Meucci N, Monaco S, Ferrari S, Bonetti B, Cavaletti G, Gemignani F, Durelli L, Barbieri S, Allaria S, Sgarzi M, et al. Frequency and clinical correlates of anti-neural $\operatorname{IgM}$ antibodies in neuropathy associated with $\operatorname{IgM}$ monoclonal gammopathy. Ann Neurol. 1994; 36: 416-424.

15. Nobile-Orazio E, Hays AP, Latov N, Perman G, Golier J, Shy ME, and Freddo L. Specificity of mouse and human monoclonal antibodies to myelin-associated glycoprotein. Neurology. 1984; 34: 1336-1342.

16. Bridoux F, Leung N, Hutchison CA, Touchard G, Sethi S, Fermand JP, Picken MM, Herrera GA, Kastritis E, Merlini G, Roussel M, Fervenza FC, Dispenzieri A, et al. Diagnosis of monoclonal gammopathy of renal significance. Kidney Int. 2015; 87: 698-711.

17. Kapoulas S, Raptis V, and Papaioannou M. New aspects on the pathogenesis of renal disorders related to monoclonal gammopathies. Nephrol Ther. 2015; 11: 135-143.

18. Turesson I, Kovalchik SA, Pfeiffer RM, Kristinsson SY, Goldin LR, Drayson MT, and Landgren O. Monoclonal gammopathy of undetermined significance and risk of lymphoid and myeloid malignancies: 728 cases followed up to 30 years in Sweden. Blood. 2014; 123: 338-345.
19. Sigurdardottir EE, Turesson I, Lund SH, Lindqvist EK, Mailankody S, Korde N, Bjorkholm M, Landgren O, and Kristinsson SY. The Role of Diagnosis and Clinical Follow-up of Monoclonal Gammopathy of Undetermined Significance on Survival in Multiple Myeloma. JAMA Oncol. 2015; 1: 168-174.

20. Gorson KC. Clinical features, evaluation, and treatment of patients with polyneuropathy associated with monoclonal gammopathy of undetermined significance (MGUS). J Clin Apher. 1999; 14: 149-153.

21. Stork AC, Lunn MP, Nobile-Orazio E, and Notermans NC. Treatment for IgG and IgA paraproteinaemic neuropathy. Cochrane Database Syst Rev. 2015; Cd005376.

22. Ramchandren $\mathrm{S}$ and Lewis RA. An update on monoclonal gammopathy and neuropathy. Curr Neurol Neurosci Rep. 2012; 12: 102-110.

23. Yeung KB, Thomas PK, King RH, Waddy H, Will RG, Hughes RA, Gregson NA, and Leibowitz S. The clinical spectrum of peripheral neuropathies associated with benign monoclonal IgM, IgG and IgA paraproteinaemia. Comparative clinical, immunological and nerve biopsy findings. J Neurol. 1991; 238: 383-391.

24. Kristinsson SY, Bjorkholm M, and Landgren O. Survival in monoclonal gammopathy of undetermined significance and Waldenstrom macroglobulinemia. Clin Lymphoma Myeloma Leuk. 2013; 13: 187-190.

25. Niermeijer JM, Fischer K, Eurelings M, Franssen H, Wokke $\mathrm{JH}$, and Notermans NC. Prognosis of polyneuropathy due to IgM monoclonal gammopathy: a prospective cohort study. Neurology. 2010; 74: 406-412.

26. Dalakas MC, Quarles RH, Farrer RG, Dambrosia J, Soueidan S, Stein DP, Cupler E, Sekul EA, and Otero C. A controlled study of intravenous immunoglobulin in demyelinating neuropathy with IgM gammopathy. Ann Neurol. 199; 40: 792-795.

27. Comi G, Roveri L, Swan A, Willison H, Bojar M, Illa I, Karageorgiou C, Nobile-Orazio E, van den Bergh P, Swan T, Hughes R, Aubry J, Baumann N, et al. A randomised controlled trial of intravenous immunoglobulin in $\mathrm{IgM}$ paraprotein associated demyelinating neuropathy. J Neurol. 2002; 249: 1370-1377.

28. Benedetti L, Briani C, Grandis M, Vigo T, Gobbi M, Ghiglione E, Carpo M, Cocito D, Caporale CM, Sormani MP, Mancardi GL, Nobile-Orazio E, and Schenone A. Predictors of response to rituximab in patients with neuropathy and anti-myelin associated glycoprotein immunoglobulin M. J Peripher Nerv Syst. 2007; 12: 102-107.

29. Leger JM, Viala K, Nicolas G, Creange A, Vallat JM, Pouget J, Clavelou P, Vial C, Steck A, Musset L, and Marin B. Placebo-controlled trial of rituximab in IgM anti-myelinassociated glycoprotein neuropathy. Neurology. 2013; 80: 2217-2225.

30. Cocito D, Paolasso I, Antonini G, Benedetti L, Briani C, Comi C, Fazio R, Jann S, Mata S, Mazzeo A, Sabatelli M, 
and Nobile-Orazio E. A nationwide retrospective analysis on the effect of immune therapies in patients with chronic inflammatory demyelinating polyradiculoneuropathy. Eur J Neurol. 2010; 17: 289-294.

31. European Federation of Neurological Societies/Peripheral Nerve Society Guideline on management of chronic inflammatory demyelinating polyradiculoneuropathy. Report of a joint task force of the European Federation of Neurological Societies and the Peripheral Nerve Society-First Revision. J Peripher Nerv Syst. 2010; 15: 1-9.

32. Simmons Z, Albers JW, Bromberg MB, and Feldman EL. Long-term follow-up of patients with chronic inflammatory demyelinating polyradiculoneuropathy, without and with monoclonal gammopathy. Brain. 1995; 118: 359-368.

33. Cesana C, Klersy C, Barbarano L, Nosari AM, Crugnola M, Pungolino E, Gargantini L, Granata S, Valentini M, and Morra E. Prognostic factors for malignant transformation in monoclonal gammopathy of undetermined significance and smoldering multiple myeloma. J Clin Oncol. 2002; 20: 1625-1634.
34. Kyle RA, Buadi F, and Rajkumar SV. Management of monoclonal gammopathy of undetermined significance (MGUS) and smoldering multiple myeloma (SMM). Oncology. (Williston Park) 2011; 25: 578-586.

35. Ludwig C, Williams DS, Bartlett DB, Essex SJ, McNee G, Allwood JW, Jewell E, Barkhuisen A, Parry H, Anandram S, Nicolson P, Gardener C, Seymour F, et al. Alterations in bone marrow metabolism are an early and consistent feature during the development of MGUS and multiple myeloma. Blood Cancer J. 2015; 5: e359.

36. Bohnhorst J, Rasmussen T, Moen SH, Flottum M, Knudsen L, Borset M, Espevik T, and Sundan A. Toll-like receptors mediate proliferation and survival of multiple myeloma cells. Leukemia. 2006; 20: 1138-1144.

37. Rajkumar SV. Prevention of progression in monoclonal gammopathy of undetermined significance. Clin Cancer Res. 2009; 15: 5606-5608. 